\title{
Lichenicolous fungi of Ukraine: an annotated checklist
}

\author{
Darmostuk VV ${ }^{1,2}$ and Khodosovtsev AYe ${ }^{1}$ \\ ${ }^{1}$ Kherson State University, 27, Universytetska str.73000, Kherson \\ ${ }^{2}$ National Nature Park «Nizhnedneprovskiy», 18, Petrenka str. 73000, Kherson
}

Darmostuk VV, Khodosovtsev AYe 2017 - Lichenicolous fungi of Ukraine: an annotated checklist. Studies in Fungi 2(1), 138-156, Doi 10.5943/sif/2/1/16

\begin{abstract}
The checklist is based on 137 references and it includes data on the distribution of 220 taxa of lichenicolous fungi in Ukraine. Four species are based on dubious records. The Crimea ( 80 species) and Carpathians Mts (64 species) have highest observed species diversity of the lichenicolous fungi. Among plain territories, most species-rich regions are Kherson (64 species) and Mykolaiv (37 species), and both regions were intensively surveyed for lichenicolous fungi. Most frequent host genera recorded are Lecanora s. lat. (23 species), Cladonia (17 species), Xanthoria s. lat. (15 species), Aspicilia s. lat. (14 species) and Caloplaca s. lat. (13 species). Most diverse taxonomical groups of lichenicolous fungi recorded are Capnodiales (21 species), Arthoniales (18 species) and Hypocreales (13 species). Katherinomyces cetrariae, Lichenochora hypanica, Lichenostigma svandae, Norrlinia medoborensis, Phoma pisutii, Pronectria diplococca, P. caloplacae, Rosellinula frustulosae, Sphaerellothecium aculeatae, Pleospora xanthoriae, Trichoconis hafellneri and Zwackhiomyces polischukii were described from type localities in Ukraine.
\end{abstract}

Key words - Arthoniales - Ascomycota - Basidiomycota - Capnodiales - host - lichen

\section{Introduction}

Lichenicolous fungi are a highly specialized group of organisms and are still poorly known. The first lichenicolous fungus recorded in Ukraine was Opegrapha physciaria (=Celidium varium (Tul.) Körb.) (Chernov 1895). During the next 100 years, lichenicolous fungi were sparsingly recorded (Oxner 1930, Servít \& Nadvornik 1932, Servít 1936, Makarevich 1955, Kopachevskaya 1986). Perheps the most substantial record of this era is the holotype specimen of Rosellinula frustulosae (Vouaux) R. Sant. on Lecanora agropholis collected by K. Mereschkowsky near Simpheropol in Crimea (Hafellner 1985). D. Hawksworth, who published nine lichenicolous fungi collected from Carpathian Mts, started the new era in discovery of these fungi in Ukraine (Hawksworth 1992). Shortly afterwards, Norrlinia medoborensis was described on Peltigera as new for science from Medobory Reserve (Ternopil region) (Kondratyuk \& Galloway 1995). Upcomming first checklist of the Ukrainian lichens (Kondratyuk et al. 1996) included information about 46 species of lichenicolous fungi. Second checklist of lichens, lichenicolous and allied fungi of Ukraine (Kondratyuk et al. 1998) listed 65 lichenicolous species. Subsequent first checklist of the lichenicolous fungi in Ukraine (Kondratyuk et al. 1999) listed 88 species with an identification key. In the last A.M. Oxner's volume of the lichen flora of Ukraine (Oxner 2010), 106 species of lichenicolous fungi were listed. In the last decade, lichenicolous fungi in Ukraine are studied with increasing intensity. Following species were recently described from Ukraine: Lichenostigma 
svadae (Vondrák \& Šoun 2007), Phoma pisutii (Oxner 2010), Pronectria diplococca, P. caloplacae (Khodosovtsev et al. 2012), Lichenochora hypanica (Kondratyuk et al. 2014), Katherinomyces cetrariae (Khodosovtsev et al. 2016), Sphaerellothecium aculeatae (Khodosovtsev et al. 2016), Pleospora xanthoriae (Khodosovtsev \& Darmostuk 2016), Trichoconis hafellneri (Braun et al. 2016) and Zwackhiomyces polischukii (Khodosovtsev \& Darmostuk 2017c). Here we provide data about 220 species of lichenicolous fungi recorded from Ukraine.

\section{Material \& Methods}

137 publications containing data on lichenicolous fungi in Ukraine were extracted; relevant references are attached to every single species in the list. Host lichen species reported for Ukrainian records are attached. Ukrainian records are sorted according to the seven geobotanical regions of Ukraine (Geobotanichne Rayonuvannya 1977): A, B: Carpathians (A: Transcarpathian lowlands, B: the mountainous part); C, D, E: Ukraine Plains (C: Forest zone, D: Forest-Steppe zone, E: Steppe zone), F, G: Crimea (F: lowlands, G: mountains). The geobotanical regions are further divided into smaller geographical units abbreviated as follows: ARC - Authonomic Republic of Crimea; $C k y-$ Cherkasy region, $C n v$ - Chernihiv region, $C v i-C h e r n i v t s i$ region, $D n-$ Dnipropetrovsk region, Do - Donetsk region, I - Ivano-Frankivsk region, Kha - Kharkiv region, Khe - Kherson region, KhmKhmelnytzky region, $K i-$ Kyiv region, Kid - Kirovograd rgion, $L k-$ Luhansk region, $L v-$ Lviv region, $M$ - Mykolaiv region, $O$ - Odesa region, $P$ - Poltava region, $R-$ Rivne region, $S$ - Sumy region, $T$ - Ternopil region, $V i$ - Vinnytzia region, $V o$ - Volyn region, Zak - Zakarpatska region, Zap - Zaporizhzhia region, $Z h-$ Zhytomyr region.

\section{An annotated checklist}

Abrothallus bertianus de Not. - on Melanelixia glabratula. B: Zak (Kondratyuk et al. 2003); C: Lv (Pirogov \& Shovhan 2015); G: ARC (Khodosovtsev et al. 2013b).

A. caerulescens I. Kotte - on Xanthoparmelia conspersa. C: Zh (Fedorenko 2006, Fedorenko et al. 2006, Kapetz 2017); E: M (Mikhailyuk et al. 2011).

A. cetrariae I. Kotte - on Platismatia glauca. B: I (Khodosovtsev et al. 2016c), Zak (Kukwa 2000).

A. parmeliarum (Sommerf.) Nyl. - on Parmelia saxatilis. B: I (Khodosovtsev et al. 2016c), Zak (Coppins et al. 1998, Kondratyuk \& al. 1998, 1999, 2003).

A. suecicus (Kirschst.) Nordin (=Vouauxiomyces ramalinae (Nordin) D. Hawksw.) - on Ramalina sp. G: $A R C$ (Kondratyuk et al. 2014).

A. teloschistis Brackel, Pérez-Ortega \& Suija - on Seirophora lacunosa. F: ARC (Khodosovtsev \& Darmostuk 2016a).

A. usneae Rabenh. - on Usnea sp. B: I (Khodosovtsev et al. 2016c).

Arthrorhaphis aeruginosa R. Sant. \& Tønsberg - on Cladonia sp. B: I (Vondrák et al. 2010).

A. grisea Th. Fr. - on Baeomyces rufus. B: Zak (Hawksworth 1992, Kondratyuk et al. 1996, 1998, 1999, 2003).

Arthonia apotheciorum (A. Massal.) Almq. (=Arthonia clemens in sense of Ukrainian authors; A. galactinaria Leight.) - on Myriolecis albescens, M. crenulata, M. dispersa, M. elenkinii, Lecanora pruinosa. B: Zak (Kondratyuk et al. 2003); C: Lv (Pirogov 2010b, 2012a); D: Khm (Kondratyuk \& Khodosovtsev 1997, Kondratyuk et al. 1999, Bielczyk et al. 2005), Cky (Kondratyuk \& Khodosovtsev 1997, Kondratyuk et al. 1999); E: Khe (Kondratyuk \& Khodosovtsev 1997, Khodosovtsev 1999a, Kondratyuk et al. 1999, Khodosovtsev 2012, Khodosovtsev \& Khodosovtseva 2014, Naumovich \& Darmostuk 2015, Darmostuk 2016a, b), $M$ (Kondratyuk \& Khodosovtsev 1997, Khodosovtsev 1999a, Kondratyuk et al. 1999), $O$ (Khodosovtsev 1999a, Khodosovtsev et al. 2016d); ARC: G (Khodosovtsev 1999a, b, 2003, 2006a, b, Fedorenko \& Akulov 2002, Khodosovtsev \& Redchenko 2002, Khodosovtsev \& Bogdan 2005, Khodosovtsev et al. 2007 as Arthonia lecanorina (Almq.) R. Sant.). 
A. epiphyscia Nyl. s.lat. - on Physconia grisea. B: I (Kondratyuk 2012); C: T (Kondratyuk \& Kolomiyets 1997, Kondratyuk et al. 1999), Zh (Kapetz 2017); G: ARC (Coppins et al. 2001, Redchenko 2001, Khodosovtsev \& Redchenko 2002).

A. lecanorina (Almq.) R. Sant - on Lecanora allophana. B: I (Khodosovtsev et al. 2016c).

A. nideri (J. Steiner) Clauzade, Diederich \& Cl. Roux - on Pyrenodesmia albopruinosa, $P$. variabilis. G: ARC (Khodosovtsev et al. 2007).

A. molendoi (Heufl. ex Frauenf.) R. Sant. - on Caloplaca sp. G: ARC (Kondratyuk et al. 1999).

A. parietinaria Hafellner \& A. Fleischhacker (=Arthonia destruens, in sense of Ukrainian authors) - on Xanthoria parietina. C: T (Kondratyuk \& Khodosovtsev 1997, Kondratyuk et al. 1999); D: Cky (Kondratyuk \& Khodosovtsev 1997, Kondratyuk et al. 1999), Kha (Kondratyuk \& Khodosovtsev 1997, Kondratyuk et al. 1999), P (Kondratyuk \& Khodosovtsev 1997), Vi (Kondratyuk \& Khodosovtsev 1997, Kondratyuk et al. 1999); E: Khe (Kondratyuk \& Khodosovtsev 1997, Kondratyuk et al. 1999, Khodosovtsev 1998, Khodosovtsev \& Khodosovtseva 2014), Ki (Kondratyuk \& Khodosovtsev 1997, Kondratyuk et al. 1999), $M$ (Kondratyuk \& Khodosovtsev 1997, Kondratyuk et al. 1999); G: ARC (Kondratyuk \& Khodosovtsev 1997). See notes (Fleischhacker et al. 2016).

A. phaeophysciae Grube \& Matzer - on Phaeophyscia endophoenicea, P. orbicularis, P. sciastra. C: Lv (Pirogov \& Khodosovtsev 2013, Pirogov \& Shovhan 2015); E: Khe (Pirogov \& Khodosovtsev 2013); G: ARC (Pirogov \& Khodosovtsev 2013).

A. punctella Nyl. s. lat. - on Circinaria calcarea. G: ARC (Khodosovtsev et al. 2007). Arthonia punctella Nyl. s.str. originally described on calcicolous Diplotomma species.

A. varians (Davies) Nyl. - on Lecanora rupicola. E: Do (Nadyeina 2005, 2009, Khodosovtsev \& al. 2013); G: ARC (Khodosovtsev 2003, 2004).

Athelia arachnoidea (Berk.) Julich - on Physcia, Phaeophyscia and Xanthoria. B: Zak (Hawksworth 1992, Coppins et al. 1998, Kondratyuk et al. 1996, 1998, 1999, 2003); C: Lv (Pirogov 2010b, 2012a), Zh (Kapetz 2017); D: Khm (Bielczyk et al. 2005), Kid (Naumovich 2009, Naumovich \& Darmostuk 2015, Khodosovtsev \& Darmostuk 2017b), Vo (Pirogov 2013); E: Dn (Naumovich \& Darmostuk 2015), Do (Khodosovtsev et al. 2013), $M$ (Boiko 2013, Darmostuk 2016d), Khe (Gavrylenko \& Khodosovtsev 2009, Gavrylenko 2011, Khodosovtsev 2012, Khodosovtsev \& Khodosovtseva 2014, 2015, Naumovich \& Darmostuk 2015, Darmostuk 2016a, e, f), Zap (Zavyalova 2010).

Biatoropsis usnearum Räsänen - on Usnea sp. G: ARC (Khodosovtsev \& Marsak 2003, Khodosovtsev et al. 2013b).

Briancoppinsia cytospora (Vouaux) Diederich, Ertz, Lawrey \& van den Boom (=Phoma cytospora (Vouaux) D. Hawksw.) - on Parmelia sulcata. B: Zak (Kondratyuk et al. 1999, 2003); C: S (Khodosovtsev \& Darmostuk 2017c); E: Khe (Khodosovtsev \& Darmostuk 2017b).

Buelliella lecanorae Suija \& Alstrup - on Lecanora chlarotera. G: ARC (Kondratyuk et al. 2014, Kapetz et al. 2015).

B. poetshii Hafellner - on Endocarpon pusillum and E. psorodeum. G: ARC (Khodosovtsev et al. 2009); E: Dn (Khodosovtsev et al. 2009, Naumovich 2009b; Naumovich \& Darmostuk 2015).

Calycina ucrainica (S.Y. Kondr.) S.Y. Kondr. (=Pezizella ucrainica S.Y. Kondr.) - on Cladonia sp. C: $T$ (Kondratyuk \& Galloway 1995, Kondratyuk et al. 1999).

Carbonea aggregantula (Müll. Arg.) Diederich \& Triebel - on Lecanora polytropa. B: Zak (Pirigov et al. 2014).

C. assimilis (Körber) Hafellner \& Hertel - on Diploschistes actinostomus. G: ARC (Khodosovtsev et al. 2007; Pirogov et al. 2014).

C. supersparsa (Nyl.) Hertel - on Lecanora sulphurea. B: Zak (Pirogov et al. 2014).

C. vitellinaria (Nyl.) Hertel - on Candelariella vitellina. B: Zak (Pirogov et al. 2014); C: Lv (Pirogov 2012a, Pirogov et al. 2014); G: ARC (Khodosovtsev 2002, Pirogov et al. 2014).

Ceratobasidium bulbillifaciens Diederich \& Lawrey - on Circinaria calcarea, Lasallia pustulata and Xanthoria parietina. E: Khe, $M$ (Khodosovtsev \& Darmostuk 2016a). 
Cercidospora caudata Kernst. s.lat. - on Caloplaca demissa. G: ARC (Khodosovtsev \& Darmostuk 2017c).

Cercidospora crozalsiana (H. Olivier) Nav.-Ros., Cl. Roux \& Casares - on Protoparmeliopsis sp. C: $Z$ h (Kapetz 2017).

C. epipolytropa (Mudd) Arnold - on Lecanora polytropa. B: I (Darmostuk 2016c, Khodosovtsev et al. 2016c), Zak (Pirigov \& Chepelevska 2013, Pirigov et al. 2015).

C. lobothalliae Nav.-Ros. \& Calat. - on Lobothallia radiosa. E: Khe (Darmostuk 2016c).

C. macrospora (Uloth) Hafellner \& Nav.-Ros. (=Cercidospora ulothii Körb.) - on Protoparmeliopsis muralis. C: Ki (Mikhailyuk et al. 2011), Zh (Fedorenko et al. 2006, 2007, Kapetz 2017); D: Cky (Fedorenko et al. 2007), Kid (Khodosovtsev \& Darmostuk 2017b); E: Dn (Naumovich \& Darmostuk 2015), Do (Darmostuk 2016c, Darmostuk \& Khodosovtsev 2014, Nadyeina 2009), Kh (Darmostuk 2017), $L k$ (Fedorenko et al. 2007), $M$ (Boiko 2010, 2013), Zap (Darmostuk 2016c, Khodosovtsev \& Zavyalova 2008a); G: ARC (Kondratyuk et al. 1999, Fedorenko et al. 2007).

C. solearispora Calat., Nav.-Ros. \& Hafellner - on Aspicilia sp. E: Zap (Darmostuk 2016c).

C. xanthoriae (Wedd.) R. Sant. - on Calogaya saxicola s.lat., Rufoplaca subpallida, Rusavskia elegans. E: $M$ (Khodosovtsev \& Darmostuk 2017c); F: ARC (Kondratyuk et al. 1999, Darmostuk 2016c).

Cladophialophora parmeliae (Etayo \& Diederich) Diederich \& Untereiner - on Xanthoparmelia conspersa. E: $M$ (Khodosovtsev \& Darmostuk 2017c).

Cladosporium licheniphilum Heuchert \& U. Braun - on Xanthoria parietina. E: Khe (Khodosovtsev \& Darmostuk 2016a).

Clypeococcum cetrariae Hafellner - on Cetraria aculeata, C. islandica. B: Zak (Pirogov 2015); C: $S$ (Khodosovtsev \& Darmostuk 2017c); E: $M$ (Khodosovtsev \& Darmostuk 2017c).

C. cladonema (Weddell) D. Hawksw. - on Cetraria islandica. C: Ki (Prekrasna et al. 2012).

C. hypocenomycis D. Hawksw. - on Carbonicola myrmecina. C: Lv (Pirogov 2010a, 2012a), Vo (Pirogov 2013), Zh (Kapetz 2017); E: Khe (Khodosovtsev \& Darmostuk 2017c).

Codonmyces lecanorae Calat. \& Etayo - on Protoparmeliopsis muralis. D: Kid (Khodosovtsev \& Darmostuk 2017b); E: Do (Darmostuk 2015a), Khe (Darmostuk 2015a, 2016b), M (Naumovich \& Darmostuk 2015).

Cornutispora ciliata Kalb - on Xanthoria parietina. E: Khe (Khodosovtsev \& Darmostuk 2016).

C. lichenicola D. Hawks. \& B. Sutt. - on Lecanora sp. B: Zak (Kondratyuk et al. 2003); C: Zh (Kapetz 2017).

C. pyramidalis Etayo - on Parmelia sulcata. C: $S$ (Khodosovtsev \& Darmostuk 2017a).

Corticifraga fuckelii (Rehm) D. Hawksw. \& R. Sant. - on Peltigera sp. B: I (Miadlikowska 1996).

Dacampia cladoniicola Halıc1 \& A.Ö. Türk - on Cladonia foliacea. E: Khe (Khodosovtsev 2011; Khodosovtsev \& Khodosovtseva 2015).

Dactylospora athallina (Müll. Arg.) Hafellner - on Baeomyces rufus. The record has not certain location (Kondratyuk et al. 1989, 1999).

D. lobariella Hafellner - on Lobaria pulmonaria. B: Zak (Raduka \& Zelenko 1998, Kondratyuk et al. 1999, 2003).

D. saxatilis (Schaer.) Hafellner (=Buellia saxatilis (Schaer.) Körb.) - on Pertusaria sp. G: ARC (Oxner 1930, Kopachevskaya 1986, Kondratyuk et al. 1999, Kondratyuk 2010).

D. rimulicola (Müll. Arg.) Hafellner - on Lecanora rupicola. G: ARC (Khodosovtsev 2011).

D. parasitica (Flörke) Arnold - on Pertusaria sp. B: Zak (Coppins et al. 1998, Kondratyuk et al. 2003).

Didymellopsis latitans (Nyl.) Clem. \& Shear - on Thallinocarpon nigrithellum. G: ARC (Khodosovtsev 2011).

D. perigena (Nyl.) Grube \& Hafellner - on Placidium squamulosum. E: Zap (Khodosovtsev \& Klymenko 2015).

D. pulposi (Zopf) Grube \& Hafellner - on Enchylium tenax. E: Khe (Khodosovtsev 2011, 2015); G: $A R C$ (Khodosovtsev 2011). 
Didymocyrtis cladoniicola (Diederich, Kocourk. \& Etayo) Ertz \& Diederich (=Phoma cladoniicola Diederich, Kocourk. \& Etayo) - on Cladonia spp. E: Dn (Naumovich \& Darmostuk 2015), Khe (Khodosovtsev \& Umanets 2009, Khodosovtsev 2012, Khodosovtsev \& Khodosovtseva 2015), $M$ (Khodosovtsev \& Umanets 2009, Darmostuk 2016d).

D. epiphyscia Ertz \& Diederich (=Phoma epiphyscia Vouaux, P. physciicola Keissl.) - on Physcia adscendens. E: Khe (Khodosovtsev 2012); G: ARC (Kondratyuk et al. 1999).

D. pseudeverniae (Etayo \& Diederich) Ertz \& Diederich - on Pseudoevernia furfuracea. B: I (Khodosovtsev et al. 2016b, c).

D. ramalinae (Roberge ex Desm.) Ertz, Diederich \& Hafellner (=Phoma ficuzzae Brackel, Pyrenidium ucrainicum S.Y. Kondr., L. Lőkös \& J.-S. Hur) - on Ramalina sp. D: Kid (Khodosovtsev \& Darmostuk 2017b); G: ARC (Khodosovtsev 2013, Kondratyuk et al. 2014).

Echinothecium reticulatum Zopf - on Evernia prinastri. G: ARC (Kondratyuk et al. 2014).

Epicladonia sandstedei (Zopf) D. Hawksw. - on Cladonia rangiformis. E: Khe (Khodosovtsev \& Darmostuk 2016a).

E. simplex D. Hawksw. - on Cladonia rangiformis. E: Khe (Khodosovtsev \& Darmostuk 2017c).

E. stenospora (Harm.) D. Hawksw. - on Cladonia foliacea. C: S (Khodosovtsev \& Darmostuk 2017a).

Erythricium aurantiacum (Lasch) D. Hawksw. \& A. Henrici (=Marchandiobasidium aurantiacum (Lasch) Diederich \& Schultheis, Marchandiomyces aurantiacus (Lasch) Diederich \& Etayo) - on Physcia, Phaeophyscia and Xanthoria. C: Zh (Kapetz 2016, 2017); D: Kid (Gavrylenko et al. 2009, Naumovich \& Darmostuk 2015, Khodosovtsev \& Darmostuk 2017b); E: Khe (Gavrylenko et al. 2009, Gavrylenko 2011, Khodosovtsev \& Khodosovtseva 2014, 2015, Naumovich \& Darmostuk 2015, Darmostuk 2016e, f); $M$ (Darmostuk 2016d).

Endococcus brachysporus (Zopf) M. Brand \& Diederich - on Porpidia tuberculosa. B: Zak (Pirigov \& Chepelevska 2015).

E. fusiger Th. Fr. \& Almq. - on Rhizocarpon distinctum. E: Zap (Khodosovtsev \& Zavyalova 2008a).

E. macrosporus (Arnold.) Nyl. - on Rhizocarpon geographicum. G: ARC (Khodosovtsev \& al. 2007).

E. propinquus (Körb.) D. Hawksw. - on Circinaria caesiocinerea. B: Zak (Servít \& Nádvorník 1932, Oxner 1956, Kondratyuk et al. 1996, 2003 as Dermatina perexigua (Arnold) Zahlbr.); C: $T$ (Smerechynska 2006, Fedorenko et al. 2007); E: $L k$ (Fedorenko et al. 2007, Nadyeina 2009, Rusina et al. 2010).

E. ramalinarius (Linds.) D. Hawksw. - on Ramalina canariensis. G: ARC (Khodosovtsev et al. 2007).

E. rugulosus Nyl. s. str. - on Verrucaria nigrescens. E: Khe (Gavrylenko \& Khodosovtsev 2009).

E. rugulosus Nyl. s. lat. - on Aspicilia cinerea, Circinaria caesiocinerea. E: Dn (Naumovich \& Darmostuk 2015), Do (Nadyeina 2009, Darmostuk 2015b), Lk (Fedorenko et al. 2007, Rusina et al. 2010), $M$ (Boiko 2008, 2013).

Halospora discrepans (J. Lahm ex Arnold) Hafellner (=Merismatium discrepans (J. Lahm ex Arnold) Triebel, Polyblastia discrepans J. Lahm ex Arnold) - on Aspicilia sp. B: Zak (Makarevich 1955, Kondratyuk et al. 1999, 2003).

Hawksworthiana peltigericola (D. Hawksw.) U. Braun - on Peltigera collina. G: ARC (Khodosovtsev et al. 2013b).

Heterocephalacria bachmannii (Diederich \& M.S. Christ.) Millanes \& Wedin (=Syzygospora bachmannii Diederich \& M.S. Christ.) - on Cladonia rangiformis. E: Khe (Khodosovtsev \& Darmostuk 2017c); G: ARC (Khodosovtsev 2013).

H. physciacearum (Diederich) Millanes \& Wedin - on Physcia stellaris. C: S (Khodosovtsev \& Darmostuk 2017a), Zh (Kapetz 2017).

Homostegia piggotii (Berk. \& Broome) P. Karst. - on Parmelia saxatilis and P. sulcata. B: I (Khodosovtsev et al. 2016c); E: Khe (Khodosovtsev \& Khodosovtseva 2015, Darmostuk 2016f); G: $A R C$ (Khodosovtsev 2013). 
Illosporiopsis christiansenii (B.L. Brady \& D. Hawksw.) D. Hawksw. (=Hobsonia christiansenii B.L. Brady \& D. Hawksw.) - on Physcia adscendens. B: Zak (Hawksworth 1992, Kondratyuk et al. 1999, 2003); C: $S$ (Khodosovtsev \& Darmostuk 2017c); E: Khe, M (Khodosovtsev \& Darmostuk 2017c).

Intralichen baccisporum P. Hawksw. \& M.S. Cole - on Acarospora cervina, Athallia pyracea, Caloplaca inconnexa, Candelariella aurella, Xanthocarpia crenulatella, X. marmorata. C: Lv (Pirogov 2012a); D: Kha (Gromakova 2014); E: Khe (Gavrylenko \& Khodosovtsev 2009, Boiko \& Khodosovtsev 2011, Naumovich \& Darmostuk 2015, Darmostuk 2016f), $M$ (Boiko $\&$ Khodosovtsev 2011, Boiko 2013), $O$ (Khodosovtsev et al. 2016d).

I. christiansenii (D. Hawksw.) D. Hawksw. \& M.S. Cole (=Bispora christiansenii D. Hawksw.) on Acarospora insolata, Athallia pyracea, Bellemerea cupreoatra, Calogaya decipiens, Caloplaca inconnexa, Candelariella aurella, Flavoplaca coronata, Lecania erysibe, Lecanora crenulata, Ramalina calicaris, Xanthocarpia crenulatella, X. marmorata. B: Zak (Hawksworth 1992, Kondratyuk et al. 1999, 2003); C: Lv (Pirogov 2012a), Zh (Kapetz 2017); D: Kid (Khodosovtsev \& Darmostuk 2017b); E: Dn (Naumovich \& Darmostuk 2015), Khe (Kondratyuk \& Khodosovtsev 1997, Gavrylenko \& Khodosovtsev 2009, Khodosovtsev \& Khodosovtseva 2014, Darmostuk 2016a), Lk (Nadyeina 2009, Rusina et al. 2010), $M$ (Boiko 2008, 2010, 2013), $O$ (Khodosovtsev et al. 2016d), Zap (Khodosovtsev \& Zavyalova 2008a).

I. lichenicola (M.S. Christ. \& D. Hawksw.) D. Hawksw. \& M.S. Cole - on Candelariella coralliza and $C$. vitellina. E: $M$ (Boiko 2010, 2013), Zap (Khodosovtsev \& Zavyalova 2008a, b).

Katherinomyces cetrariae Khodos. - on Cetraria aculeata. E: Khe (Khodosovtsev et al. 2016e).

Knufia peltigerae (Fuckel) Réblová \& Unter. (=Capronia peltigerae (Fuckel) D. Hawksw.) - on Peltigera rufescens. C: T (Kondratyuk \& Kolomiyets 1997, Kondratyuk et al. 1999, Smerechynska 2006).

Laetisaria lichenicola Diederich, Lawrey \& Van den Broeck - on Physcia adscendens. E: Khe (Khodosovtsev \& Darmostuk 2017c).

Libertiella malmedyensis Speg. \& Roum. - on Peltigera rufescens. B: Cvi (Kondratyuk et al. 1999); C: $T$ (Kondratyuk \& Kolomiyets 1997, Kondratyuk et al. 1999).

Lichenochora caloplacae Zhurb. - on Athallia skii. E: Khe (Khodosovtsev \& Darmostuk 2017c).

L. hypanica S. Y. Kondr., L. Lőkös \& J.-S. Hur - on Endocarpon obscuratum. E: M (Kondratyuk et al. 2014).

L. obscuroides (Linds.) Triebel \& Rambold - on Phaeophyscia spp. B: I (Kondratyuk 2012, Kapetz et al. 2015); C: $L v$ (Pirogov 2012a), $R$ (Kapetz et al. 2015), $Z h$ (Kapetz et al. 2015, Kapetz 2017); D: $C k y$ (Kapetz et al. 2015).

L. weillii (Werner) Hafellner \& R. Sant. - on Physconia grisea. E: Khe (Khodosovtsev \& Darmostuk 2017c).

Lichenoconium erodens M. S. Christ. \& D. Hawksw. - on Cladonia fimbriata, Evernia prunastri, Hypogymnia physodes, Lecanora argentata, L. conizaeoides, Parmelia submontana, Pleurosticta acetabulum, Ramalina farinacea, Xanthoparmelia conspersa, X. pulla. B: Zak (Hawksworth 1992, Motiejūnaite et al. 1999, Kondratyuk et al. 1999, 2003); C: Lv (Pirogov 2012a), Vo (Pirogov 2013), Zh (Kapetz 2017); E: Khe (Khodosovtsev \& Khodosovtseva 2015), $M$ (Boiko 2013, Darmostuk 2016d, Khodosovtsev \& Darmostuk 2017), Zap (Zavyalova 2010); D: Kid (Khodosovtsev \& Darmostuk 2017b); G: ARC (Kondratyuk et al. 2014).

L. lecanorae (Jaap) D. Hawksw - on Hypogymnia physodes, Lecanora carpinea, L. conizaeoides, Parmeliopsis ambigua, Protoparmeliopsis muralis. B: I (Khodosovtsev et al. 2016c), Zak (Motiejūnaitė et al. 1999, Kondratyuk et al. 1999, 2003, Fedorenko et al. 2007); C: Lv (Pirogov 2012a), Zh (Fedorenko et al. 2006, 2007, Kapetz 2017); E: Khe (Khodosovtsev 2012, Darmostuk 2016b, f), $M$ (Naumovich \& Darmostuk 2015), $O$ (Khodosovtsev et al. 2016d); G: ARC (Khodosovtsev 2013).

L. pyxidatae (Oudem.) Petrak et H. Sydow - on Cladonia foliacea. E: Khe (Khodosovtsev 2011). 
L. usneae (Anzi) D. Hawksw. - on Physcia stellaris. B: Zak (Hawksworth 1992, Kondratyuk et al. 1999, 2003); C: Zh (Kapetz 2017).

L. xanthoriae M.S. Christ - on Xanthoria parietina. C: Lv (Pirogov 2012a), T (Kondratyuk \& Kolomiyets 1997, Kondratyuk et al. 1999, Smerechynska 2006); E: Khe (Gavrylenko 2011, Darmostuk 2016f), $M$ (Boiko 2013).

Lichenodiplis lecanorae (Vouaux) Dyko \& D. Hawksw - on Lecanora saligna. C: Vo (Pirogov 2013), Zh (Kapetz 2016, 2017); E: Khe (Khodosovtsev \& Khodosovtseva 2014, 2015, Naumovich \& Darmostuk 2015, Darmostuk 2016b, e), Lk (Nadyeina 2009), Zap (Kondratyuk et al. 1999).

Lichenohendersonia varians Calatayud \& Etayo - on Candelariella vitellina. E: Zap (Khodosovtsev \& Darmostuk 2016a).

Lichenopeltella peltigericola (D. Hawksw.) R. Sant. - on Peltigera sp. B: I (Miadlikowska 1996, Kondratyuk et al. 1999, 2003).

Lichenostigma cosmopolites Hafellner \& Calatayud - on Xanthoparmelia conspersa. B: I (Khodosovtsev et al. 2016c), Zak (Kondratyuk et al. 2003, Kondratyuk 2005, Fedorenko \& al. 2007); C: $Z$ h (Fedorenko et al. 2006, 2007, Kapetz 2017); D: Kid (Khodosovtsev \& Darmostuk 2017b); E: Dn (Naumovich 2009b, Naumovich \& Darmostuk 2015), Do (Khodosovtsev et al. 2013), $L k$ (Fedorenko et al. 2007, Nadyeina 2009, Rusina et al. 2010), $M$ (Boiko 2013), Zap (Khodosovtsev \& Zavyalova 2008a); G: ARC (Kondratyuk 2005, Fedorenko et al. 2007).

L. elongatum Nav.-Ros. \& Hafellner - on Aspicilia spp., Lobothallia radiosa and Prothoparmeliopsis muralis. B: Zak (Coppins et al. 1998, Kondratyuk et al. 1999, 2003, Kondratyuk 2005); D: Kid (Khodosovtsev \& Darmostuk 2017b); E: Dn (Naumovich \& Darmostuk 2015), Do (Nadyeina 2009, Khodosovtsev et al. 2013, Darmostuk \& Khodosovtsev 2014), Khe (Gavrylenko \& Khodosovtsev 2009, Naumovich \& Darmostuk 2015, Darmostuk 2016a, b), $M$ (Boiko 2008, 2013, Naumovich \& Darmostuk 2015), $O$ (Khodosovtsev \& al. 2016d); G: ARC (Kondratyuk 2005, Vondrák \& Šoun 2007).

L. epipolina Nav.-Ros., Calat. \& Hafellner - on Diplotomma epipolium. C: Zh (Kapetz 2017).

L. gracilis Calat., Nav.-Ros. \& Hafellner - on Acarospora fuscata. B: I (Khodosovtsev et al. 2016c); E: Do (Khodosovtsev et al. 2013); G: ARC (Kondratyuk 2005).

L. maureri Hafellner - on Pseudoevernia furfuracea. B: I (Khodosovtsev et al. 2016c), Zak (Hawksworth 1992, Kondratyuk et al. 1999, 2003); C: S (Khodosovtsev \& Darmostuk 2017c); E: Khe, $M$ (Khodosovtsev \& Darmostuk 2017c); G: ARC (Khodosovtsev et al. 2013b).

L. rouxii Nav.-Ros., Calat. \& Hafellner - on Squamarina cartilaginea. G: ARC (Kondratyuk 2005, Vondrák \& Šoun 2007).

L. rugosa G. Thor - on Diploschistes candidissimus. E: Khe (Gavrylenko 2010, 2012).

L. svandae Vondrák \& Šoun - on Acarospora cervina. E: Khe (Gavrylenko \& Khodosovtsev 2009), $M$ (Boiko 2013, Naumovich \& Darmostuk 2015); G: ARC (Vondrák \& Šoun 2007).

Lichenothelia convexa Henssen - on Acarospora sp., Circinaria caesiocinerea, Rhizocarpon geographicum. B: Zak (Fedorenko et al. 2007); C: Zh (Kapetz 2017); D: Kid (Khodosovtsev \& Darmostuk 2017b); E: Lk (Fedorenko et al. 2007, Nadyeina 2009); G: ARC (Khodosovtsev 2004, Fedorenko et al. 2007).

L. scopularia (Nyl.) D. Hawksw. - on Aspicilia sp. and siliceous rocks. C: Zh (Fedorenko et al. 2007; Kapetz 2017); G: ARC (Khodosovtsev et al. 2007).

L. renobalesiana D. Hawksw. \& V. Atienza - on Bagliettoa sp. G: ARC (Khodosovtsev \& Darmostuk 2016a).

L. tenuissima Henssen - on Lecidea fuscoatra, Prothoparmelia sp., Scoliciosporum sp. C: Zh (Kapetz 2016, 2017).

Llimoniella groenlandiae (Alstrup \& D. Hawksw.) Triebel \& Hafellner (=Llimoniella caloplacae S. Kondr. \& Khodos.) - on Xanthocarpia borysthenica. E: Khe (Kondratyuk et al. 2006, Khodosovtsev 2015). 
Marchandiomyces corallinus (Roberge) Diederich \& D. Hawksw - on Physconia grisea. C: Zh (Kapetz 2017); E: Khe (Khodosovtsev \& Khodosovtseva 2014).

Microsphaeropsis caloplacae Etayo \& Yazıc1 - on Calogaya lobulata. E: Khe (Khodosovtsev \& Darmostuk 2017c).

Milospium graphideorum (Nyl.) D. Hawksw. - on Dirina stenhammari. B: Zak (Vondrák et al. 2010); G: $A R C$ (Khodosovtsev et al. 2007).

M. lacoizquetae Etayo \& Diederich - on Cladonia sp. B: I (Khodosovtsev et al. 2016b, c).

Monodictys epilepraria Kukwa \& Diederich - on Lepraria sp. B: Zak (Vondrák et al. 2010); C: Zh (Kapetz 2016, 2017).

Muellerella lichenicola (Sommerf.) D. Hawksw. - on Acarospora cervina, Candelariella oleifera, Circinaria calcarea, Lecanora albescens, L. crenulata, L. dispersa, Rinodina bischoffii, Protoblastenia rupestris, Variospora dolomitica, Verrucaria sp. C: Lv (Pirogov 2010a, 2012a), T (Smerechynska 2006, Fedorenko et al. 2007); D: Khm (Bielczyk et al. 2005); E: Khe (Kondratyuk \& Khodosovtsev 1997, Kondratyuk et al. 1999, Naumovich \& Darmostuk 2015, Darmostuk 2016f), $L k$ (Fedorenko et al. 2007, Nadyeina 2009), $M$ (Boiko 2008, 2013, Naumovich \& Darmostuk 2015), $O$ (Khodosovtsev et al. 2016b); G: ARC (Kondratyuk \& Khodosovtsev 1997, Kondratyuk et al. 1999, Khodosovtsev 1999, Khodosovtsev \& Redchenko 2002, Fedorenko et al. 2007).

M. hospitans Stizenb - on Bacidia fraxinea. B: Zak (Kukwa 2000, Kondratyuk et al. 2003); G: ARC (Khodosovtsev 2000).

M. pygmaea (Körb.) D. Hawksw. - on Acarospora cervina, Arthonia lapidicola, Bellemeria cupreoatra, Candelariella sp., Lobothallia alphoplaca, Rufoplaca arenaria. B: I (Kondratyuk 2012); C: T (Kondratyuk \& Khodosovtsev 1997, Kondratyuk et al. 1999, Smerechynska 2006, Fedorenko et al. 2007), Zh (Kapetz 2017); D: Khm (Bielczyk et al. 2005), Kid (Khodosovtsev \& Darmostuk 2017b); E: Dn (Naumovich \& Darmostuk 2015), Khe (Kondratyuk \& Khodosovtsev 1997), Lk (Fedorenko et al. 2007, Nadyeina 2009, Rusina et al. 2010), M (Boiko 2013), Zap (Khodosovtsev \& Zavyalova 2008a); G: ARC (Kondratyuk \& Khodosovtsev 1997, Kondratyuk et al. 1999, Khodosovtsev 1999, Fedorenko et al. 2007).

M. ventosicola (Mudd) D. Hawksw. - on Rhizocarpon geographicum. B: I (Khodosovtsev et al. 2016c), Zak (Nadyeina \& Halıc1 2011); E: Zap (Nadyeina \& Halıc1 2011).

Nectriopsis lecanodes (Ces.) Diederich \& Schroers (=Nectria lecanodes (Ces.) Fr.) - on Peltigera canina and P. horizontalis. C: Lv (Pirogov 2012a), unspecified location (Morochkovskiy et al. 1969, Kondratyuk et al. 1999).

N. rubefaciens (Ellis \& Everh.) M.S. Cole \& D. Hawksw. - on Parmelia sulcata. C: Lv (Pirogov 2011, 2012a); E: Khe (Khodosovtsev \& Darmostuk 2017c).

Norrlinia medoborensis S. Kondr. - on Peltigera rufescens and P. praetextata. C: $T$ (Kondratyuk \& Galloway 1995, Kondratyuk et al. 1999, Smerechynska 2006).

Opegrapha centrifuga A. Massal. - on Verrucaria nigrescens. E: Khe (Gavrylenko 2012), O (Khodosovtsev et al. 2016b).

O. parasitica (A.Massal.) H. Olivier - on Leproplaca cirrochroa. G: ARC (Khodosovtsev 2006c).

O. physciaria (Nyl.) D. Hawksw. \& Coppins (=Celidium varium (Tul.) Körb.) - on Physcia adscendens, Xanthoria parietina. C: Lv (Kondratyuk \& Khodosovtsev 1997, Kondratyuk \& al. 1999, Pirogov 2012a); D: Kha (Chernov 1895, Kondratyuk et al. 1999 as Arthonia molendoi); E: Khe (Kondratyuk \& Khodosovtsev 1997, Kondratyuk et al. 1999, Khodosovtsev \& Khodosovtseva 2014) G: ARC (Kondratyuk \& Khodosovtsev 1997, Kondratyuk et al. 1999).

O. pulvinata Rehm ap Lojka - on Dermatocarpon miniatum. B: Zak (Kondratyuk et al. 1999, 2003).

O. rupestris Pers. - on Pyrenodesmia variabilis, Verrucaria sp. B: Zak (Kondratyuk et al. 2003); C: $Z h$ (Fedorenko et al. 2006); D: Khm (Bielczyk et al. 2005); E: $O$ (Khodosovtsev et al. 2016b); G: ARC (Khodosovtsev \& Bogdan 2005, Khodosovtsev 2006d). 
O. verrucariae Coppins, S. Y. Kondr. \& Etayo - on Verrucaria sp. G: ARC (Kondratyuk et al. 2014).

Paranectria oropensis (Cesati in Rabenh.) D. Hawksw. \& Piroz. - on Lecanora carpinea. B: I (Khodosovtsev et al. 2016c), Zak (Coppins et al. 1998, Kondratyuk et al. 1999, 2003).

Phacographa zwackhii (A. Massal. Ex Zwackh.) Hafellner - on Phlyctis argena. G: ARC (Khodosovtsev et al. 2013b).

Phaeospora lecanorae Eitner - on Lecanora dispersa. G: ARC (Khodosovtsev et al. 2007).

P. rimosicola (Leight. ex Mudd) Hepp ex Stein - on Rhizocarpon petraeum. B: Zak (Pirogov \& Sharavara 2012).

Phoma lobariae Etayo \& Diederich - on Lobaria pulmonaria. B: Zak (Kondratyuk et al. 2003).

P. pisutii S.Y. Kondr., Lackovičová, Lisická \& Guttová - on Xanthoria parietina. B: I (Kondratyuk 2012).

Plectocarpon lichenum (Sommerf.) D. Hawksw. - on Lobaria pulmonaria. G: ARC (Khodosovtsev et al. 2013b).

Pleospora xanthoriae Khodos. \& Darmostuk - on Xanthoria parietina. E: Khe (Khodosovtsev \& Darmostuk 2016b).

Polycoccum aksoyi Halıc1 \& V. Atienza - on Aspicilia sp. E: Dn (Darmostuk \& Golovenko 2016).

P. marmoratum (Krempelh.) D. Hawksw. (=Microthelia marmorata (Kremp.) Hepp) - on Bagliettoa sp. E: Khe (Gavrylenko \& Khodosovtsev 2009); G: ARC (Kopachevskaya 1986, Kondratyuk et al. 1999).

P. microcarpum Diederich \& Etayo - on Cladonia sp. B: Zak (Flakus et al. 2008).

P. microsticticum (Leight. ex Mudd) Arnold - on Acarospora fuscata. C: Zh (Fedorenko et al. 2006, 2007).

P. pulvinatum (Eitner) R. Sant. (=Polycoccum galligeum Vezda) - on Physcia caesia. C: T (Kondratyuk \& Khodosovtsev 1997, Kondratyuk et al. 1999, Fedorenko 2006); Zh (Fedorenko 2006, Fedorenko et al. 2007); D: Kid (Khodosovtsev \& Darmostuk 2017b); E: $M$ (Boiko 2013).

P. teresum Halıc1 \& K. Knudsen - on Xanthoria calcicola. G: ARC (Khodosovtsev 2011).

Polysporina subfuscescens (Nyl.) K. Knudsen \& Kocourk. (=Polysporina lapponica auct.) - on Acarospora, Buellia, Caloplaca and Candelariella. C: Zh (Kapetz et al. 2015, Kapetz 2017); C: Zap (Khodosovtsev \& Zavyalova 2008b).

Pronectria caloplacae Khodos., J. Vondrák \& A. Naumovich - on Flavoplaca austrocitrina. E: Khe (Khodosovtsev et al. 2012).

P. casaresii Etayo - on Evernia prunastri. E: Khe (Khodosovtsev \& Darmostuk 2017c).

P. $\boldsymbol{c f}$. dillmaniae Zhurb. - on Placidium squamulosum. E: Khe (Khodosovtsev \& Darmostuk 2017c).

P. diplococca Kocourk., Khodos., Naumovich, Vondrák \& Motiej. - on Blennothallia crispa. D: P (Khodosovtsev \& Darmostuk 2017c); E: Khe (Khodosovtsev et al. 2012, Khodosovtsev 2015).

P. robergei (Mont. \& Desmaz.) Lowen (=Nectria lichenicola (Ces.) Sacc.) - on Peltigera canina, P. didactyla, Cladonia sp. C: Lv (Pirogov 2012a), unspecified location (Morochkovskiy et al. 1969, Kondratyuk et al. 1999).

P. fissuriproidens Etayo - on Lobaria pulmonaria. B: Zak (Kondratyuk et al. 2003).

P. oligospora Etayo - on Punctelia sp. B: Zak (Kondratyuk et al. 2003).

Psammina stipitata D. Hawksw. - on algae crust on wood. B: I (Khodosovtsev et al. 2016b, c).

Pyrenidium actinellum Nyl. - on Aspicilia cinerea, Cladonia chlorophaea. B: Zak (Pirogov 2014); C: $T$ (Kondratyuk et al. 1999); E: Dn (Naumovich 2009a, b, Naumovich \& Darmostuk 2015).

Pyrenochaeta xanthoriae Diederich - on Xanthoria parietina. C: Zh (Kapetz 2017); E: Khe (Khodosovtsev 2010, 2012), $M$ (Darmostuk 2016d).

Refractohilum intermedium $\mathrm{Cl}$. Roux \& Etayo - on Pachyphiale arbuti and P. carneola. C: $S$ (Khodosovtsev \& Darmostuk 2017a); E: Khe (Khodosovtsev \& Darmostuk 2017a); G: ARC (Khodosovtsev \& Darmostuk 2017a). 
Reichlingia leopoldii Diederich \& Scheidegger - on Lobaria pulmonaria. B: Zak (Coppins et al. 1998; Kondratyuk et al. 1999, 2003).

Roselliniella cladoniae (Anzi) Matzer \& Hafellner - on Cladonia sp. D: Khm (Bielczyk et al. 2005).

R. frustulosae (Vouaux) R. Sant. - on Lecanora agropholis. G: ARC (Hafellner 1985).

Sagediopsis barbata (Th. Fr.) R. Sant. \& Triebel - on Porpidia sp. B: Zak (Servít 1936, Coppins et al. 1998, Kondratyuk et al. 1999, 2003).

Sarcopyrenia cylindrospora (P. Crouan \& H. Crouan) M.B. Aguirre - on Aspicilia sp. E: Zap (Khodosovtsev \& Zavyalova 2008b).

Sarocladium strictum (W. Gams) Summerb. (=Acremonium strictum W. Gams) - on Physcia stellaris. B: Zak (Hawksworth 1992, Kondratyuk et al. 1999, 2003).

Sclerococcum griseisporodochium Etayo - on unknown lichen. B: Zak (Vondrák et al. 2010).

S. sphaerale (Ach.) Fr. - on Pertusaria sp. C: Zh (Kapetz 2016); E: Zap (Kondratyuk et al. 1999).

Scutula epiblastematica (Wallr.) Rehm - on Peltigera rufescens. B: Zak (Kondratyuk et al. 2003); C: Khm (Kondratyuk \& Kolomiyets 1997), Lv (Pirogov 2012a), T (Kondratyuk et al. 1999, Smerechynska 2006).

S. tuberculosa (Th. Fr.) Rehm - on Peltigera rufescens. C: T (Kondratyuk \& Kolomiyets 1997 , Kondratyuk et al. 1999, Smerechynska 2006).

Skyttea acrocordiae Diederich - on Acrocordia sp. B: Zak (Kondratyuk et al. 2003).

Sphaerellothecium aculeatae Khodos., Gavrylenko \& Klymenko - on Cetraria aculeata. E: Khe (Khodosovtsev et al. 2016e).

S. parmeliae Diederich \& Etayo - on Parmelia saxatilis. B: I (Khodosovtsev et al. 2016c); G: ARC (Khodosovtsev 2013).

S. parietinarium (Linds.) Hafellner \& V. John (=Endococcus parietinarius (Linds.) D. Hawksw.) on Xanthoria parietina. C: Lv (Pirogov 2012a), Vo (Pirogov 2013); G: ARC (Kondratyuk \& al. 1999).

S. propinquellum (Nyl.) Cl. Roux \& Triebel - on Lecanora sp. (Subfusca group). C: Zh (Kapetz 2017); G: ARC (Khodosovtsev 2013).

Sphinctrina anglica Nyl. - on Protoparmelia sp. B: Cvi (Makarevich et al. 1982, Kondratyuk et al. 1999), Zak (Makarevich 1955, Kondratyuk et al. 2003).

S. tubiformis A. Massal. - on Pertusaria sp. G: ARC (Coppins et al. 2001, Khodosovtsev 2003).

S. turbinata (Pers.) de Not. - on Pertusaria sp. B: Cvi (Kondratyuk et al. 1999), Zak (Kondratyuk et al. 1999, 2003, Pirogov et al. 2015); C: Ki (Kondratyuk et al. 1999); Zh (Kondratyuk et al. 1999, Fedorenko et al. 2006); D: Chy (Kondratyuk et al. 1999); G: ARC (Kopachevskaya 1986, Kondratyuk et al. 1999, Khodosovtsev \& Bogdan 2005, Khodosovtsev et al. 2013b).

Stigmidium clauzadei Cl. Roux \& Nav.-Ros. - on Verrucaria viridula. E: Khe (Khodosovtsev \& Darmostuk 2016a).

S. congestum (Körb.) Triebel - on Lecanora chlarothera. E: M (Naumovich \& Darmostuk 2015); G: $A R C$ (Khodosovtsev et al. 2013b).

S. conspurcans (Th. Fr.) Triebel \& R. Sant. - on Psora testacea. G: ARC (Kondratyuk et al. 1999).

S. glebarum (Arnold) Hafellner - on Toninia physaroides. E: $M$ (Boiko 2008, 2013, Boiko \& Khodosovtsev 2011); $O$ (Khodosovtsev et al. 2016b).

S. gyrophorarum (Arnold) D. Hawksw. - on Umbilicaria cylindrica. B: I (Darmostuk \& Gavrylenko 2016), Zak (Darmostuk \& Gavrylenko 2016).

S. fuscatae (Arnold) R. Sant. - on Acarospora fuscata. B: I (Khodosovtsev et al. 2016c); C: Zh (Fedorenko 2006, Fedorenko et al. 2006, Kapetz 2017); E: $M$ (Mikhailyuk et al. 2011).

S. lichenum (Arnold) Triebel \& P. Scholz (=Pharcidia lichenum Arnold) - on Verrucaria sp. C: unspecified location (Morochkovskiy et al. 1969, Kondratyuk et al. 1999 as Stigmidium dispersum (Lahm ex Korb.) D. Hawksw.).

S. microspilum (Körb.) D. Hawksw. (=Arthopyrenia microspila Körb.) - on Graphis scripta. B: Zak (Hawksworth 1992, Coppins et al. 1998, Kondratyuk et al. 1999, 2003); C: T (Kondratyuk \& Kolomiyets 1997, Kondratyuk et al. 1999, Smerechynska 2006). 
S. mycobilimbiae $\mathrm{Cl}$. Roux, Triebel \& Etayo - on Mycobilimbia sabuletorum. E: $O$ (Khodosovtsev et al. 2016b).

S. peltideae (Vainio) R. Sant. - on Peltigera sp. B: Zak (Miadlikowska 1996, Kondratyuk et al. 1999, 2003).

S. pumilum (Lettau) Matzer \& Hafellner - on Physcia caesia. C: Zh (Fedorenko et al. 2006, 2007).

S. rouxianum Calatayud \& Triebel - on Acarospora cervina. C: Khm (Bielczyk et al. 2005); E: Khe (Naumovich 2009a); G: ARC (Khodosovtsev et al. 2007).

S. tabacinae (Arnold) Triebel - on Toninia sedifolia. C: Khm (Bielczyk et al. 2005).

S. squamariae (B. de Lesd.) Cl. Roux \& Triebel - on Prothoparmeliopsis muralis. B: Zak (Pirogov et al. 2015); C: $L v$ (Pirogov 2012a); E: $M$ (Khodosovtsev \& Darmostuk 2017c).

S. xanthoparmeliarum Hafellner - on Xanthoparmelia conspersa. C: Zh (Kapetz 2017); D: Kid (Khodosovtsev \& Darmostuk 2017b); E: Dn (Naumovich \& Darmostuk 2015).

Taeniolella beschiana Diederich - on Cladonia sp. C: Zh (Kapetz 2017).

T. delicata M.S. Christ. \& D. Hawksw. - on Myriolecis crenulata. C: T (Kondratyuk \& Kolomiyets 1997, Kondratyuk et al. 1999, Smerechynska 2006).

T. friesii (Hepp) Hafellner - on Strigula stigmatella. B: Zak (Kondratyuk et al. 2003).

T. phaeophysciae D. Hawksw. - on Phaeophyscia orbicularis. B: Zak (Kondratyuk et al. 2003); C: $L v$ (Pirogov \& Khodosovtsev 2013), $R$ (Pirogov \& Khodosovtsev 2013), Zh (Kapetz 2017); E: Khe (Pirogov \& Khodosovtsev 2013, Khodosovtsev \& Khodosovtseva 2014, 2015); G: ARC (Pirogov \& Khodosovtsev 2013).

T. punctata M. S. Christ. \& D. Hawksw. - on Graphis scripta. B: Zak (Kondratyuk et al. 2003); C: Khm (Bielczyk et al. 2005), Zh (Kapetz et al. 2015, Kapetz 2017).

Telogalla olivieri (Vouaux) Nik. Hoffm. \& Hafellner (=Guignardia olivieri (Vouaux) Olivier) - on Xanthoria parietina. B: I (Navrotskaya et al. 1996, Kondratyuk 2012), Zak (Kondratyuk et al. 2003); E: Khe (Navrotskaya et al. 1996).

Toninia talparum Timdal - on Lecania erysibe and L. inundata. D: Khm (Zelenko 2005).

T. subfuscae (Arnold) Timdal - on Verrucaria nigrescens. E: $O$ (Khodosovtsev et al. 2016b).

Tremella cladoniae Diederich \& M.S. Christ. - on Cladonia sp. B: Zak (Motiejūnaitè et al. 1999, Kondratyuk et al. 1999, 2003).

T. hypogymniae Diederich \& M.S. Christ - on Hypogymnia physodes. B: Zak (Motiejūnaitè et al. 1999, Kondratyuk et al. 2003); G: ARC (Khodosovtsev et al. 2013b).

T. lichenicola Diederich - on Mycoblastus fucatus. B: Zak (Coppins et al. 1998, Kondratyuk et al. 1999, 2003, Vondrák et al. 2010).

T. phaeophysciae Diederich \& M.S. Christ. - on Phaeophyscia orbicularis. B: I (Kondratyuk 2012); E: $M$ (Khodosovtsev \& Darmostuk 2017c).

Trichoconis hafellneri U. Braun, Khodos., Darmostuk \& Diederich - on Athallia pyracea and Xanthoria parietina. D: $P$ (Braun et al. 2016); E: Khe (Braun et al. 2016).

Trichonectria anisospora (Lowen) van der Boom \& Diederich - on Hypogymnia physodes. C: Lv (Pirogov 2012b).

T. hirta (A. Bloxam) Petch. - on Scoliciosporum chlorococcum. B: Zak (Kondratyuk et al. 2003); C: $L v$ (Pirogov 2010b, 2012a), Zh (Kondratyuk et al. 1999, Fedorenko \& al. 2007, Kapetz 2017).

Unguiculariopsis thallophila (P. Karst.) W.Y. Zhuang - on Prothoparmeliopsis muralis. E: Dn (Darmostuk \& Naumovich 2016).

Vouauxiella lichenicola (Linds.) Petr. \& Syd. - on Lecanora chlorotera. B: Zak (Kukwa 2000, Pirogov \& Sharavara 2013); C: Vo (Pirogov \& Sharavara 2013).

V. verrucosa (Vouaux) Petr. \& Syd. - on Lecanora argentata. G: ARC (Khodosovtsev \& Darmostuk 2016a).

Weddellomyces heterochrous Nav.-Ros. \& Cl. Roux - on Aspicilia sp. E: Lk (Nadyeina \& Halıc1 2012).

Xanthoriicola physciae (Kalchbr.) D. Hawksw. - on Xanthoria parietina. B: I (Kondratyuk 2012), Zak (Coppins et al. 1998, Kondratyuk et al. 2003); C: Khm (Bielczyk et al. 2005), Lv 
(Pirogov 2012a, Pirogov \& Shovhan 2015), $T$ (Kondratyuk \& Kolomiyets 1997, Kondratyuk et al. 1999, Smerechynska 2006), Vo (Pirogov 2013), Zh (Fedorenko et al. 2006, Kapetz 2017); D: Kha (Gromakova 2014); E: Khe (Khodosovtsev 2012, Khodosovtsev \& Khodosovtseva 2014, 2015, Naumovich \& Darmostuk 2015, Darmostuk 2016a, e, f), M (Boiko 2013, Darmostuk 2016d), Zap (Zavyalova 2010).

Xenonectriella leptaleae (J. Steiner) Rossman \& Lowen - on Phaeophyscia orbicularis and Physcia stellaris. C: Lv (Pirogov 2012b), Zh (Kapetz 2017); S (Khodosovtsev \& Darmostuk 2017c); E: $M$ (Khodosovtsev \& Darmostuk 2017c).

Zwackhiomyces berengerianus (Arnold) Grube \& Triebel - on Mycobilimbia sabuletorum. B: Zak (Khodosovtsev \& Darmostuk 2016a); E: $O$ (Khodosovtsev \& Darmostuk 2016a).

Z. calcariae (Flagey) Hafellner \& Nik. Hoffm. - on Circinaria calcarea. E: M (Boiko 2008, 2013, Boiko \& Khodosovtsev 2011).

Z. cervinae Calat., Triebel \& Pérez-Ortega - on Acarospora cervina. G: ARC (Khodosovtsev \& Klymenko 2015).

Z. coepulonus (Norman) Grube \& R. Sant. - on Xanthocarpia crenulatella. C: Khm (Kondratyuk \& Kolomiyets 1997), T (Kondratyuk \& Kolomiyets 1997, Kondratyuk et al. 1999, Smerechynska 2006), Zh (Kondratyuk \& Kolomiyets 1997); E: Do (Nadyeina 2009), Khe (Gavrylenko \& Khodosovtsev 2009), Lk (Nadyeina 2009), M (Khodosovtsev et al. 2007, Boiko 2013); G: ARC (Khodosovtsev et al. 2007).

Z. diederichii D. Hawksw. \& Ittur. - on Cladonia rangiformis. E: Khe (Khodosovtsev 2011).

Z. dispersus (J. Lahm et Körb.) Triebel et Grube - on Protoblastenia rupestris. G: ARC (Kondratyuk et al. 1999, Khodosovtsev 2011).

Z. lecanorae (Stein) Nik. Hoffman \& Hafellner - on Myriolecis semipallida, Prothoparmeliopsis muralis. C: Khm (Bielczyk et al. 2005); E: Khe (Gavrylenko 2014, Naumovich \& Darmostuk 2015, Darmostuk 2016b), $M$ (Naumovich \& Darmostuk 2015), $O$ (Khodosovtsev et al. 2016b).

Z. polischukii Darmostuk \& Khodos. - on Bacidia fraxinea and B. rubella. C: Khm (Khodosovtsev \& Darmostuk 2017c); G: ARC (Khodosovtsev \& Darmostuk 2017c).

Z. sphinctrinoides (Zwackh) Grube \& Hafellner - on Lecanora campestris. G: ARC (Kondratyuk et al. 1999).

\section{Dubious records}

Lichenoconium lichenicola (P. Karsten) Petrak \& H. Sydow - on Hypogymnia physodes and Flavoparmelia caperata. C: Ki (Gizhytska 1929 as Coniothyrium lichenicola P. Karst., Kondratyuk et al. 1999). The record probably belongs to a different species because $L$. lichenicola only occurs on Physcia species (Lawrey et al. 2011).

Phoma denigricans Hafellner - on Protoparmelia sp. E: Zap (Kondratyuk et al. 1999). Respective specimen (KHER 890) is characterized by immersed black subspherical pycnidia, 80-100 $\mu \mathrm{m}$ diam, ampulliform conidiogenous cells, 4-5.5 × 2.5-4 $\mu \mathrm{m}$, and ellipsoid conidia (4.5)5.0-6.25-7.5(-9.5) $\times(2.25-) 2.75-3.25-3.75(-4.25) \mu \mathrm{m}[\mathrm{n}=30], 1 / \mathrm{b}$ ratio (1.5-)2.0-2.4-2.8(3.1). The specimen is morphologicaly similar to Didymocyrtis foliaceiphila (Diederich, Kocourk. \& Etayo) Ertz \& Diederich (Ertz et al. 2015).

Polycoccum bryonthae (Arnold) Vězda - on Lecanora epibryon. G: ARC (Kondratyuk et al. 1999). This specimen needs future confirmation, because the host, Lecanora epibryon, is not known from Ukraine (Oxner 2010).

Stigmidium schaereri (A. Massal.) Trevis. - on Lecanora sp. E: $M$ (Kondratyuk \& Khodosovtsev 1997, Kondratyuk et al. 1999, Boiko 2013); G: ARC (Redchenko 2001, Khodosovtsev \& Redchenko 2002). Records from Ukraine need revision, because $S$. schaereri is lichenicolous only on Solorina spp. (Roux \& Triebel 1994). 


\section{Acknowledgements}

We express our gratitude to Jan Vondrák (Czech Republic) who commented the manuscript. This study was financially supported by the project of Ministry of Science and Education of Ukraine N 0116 U004735.

\section{References}

Bielczyk U, Bylinska E, Czarnota P, Czyzewska K et al. 2005 - Contribution to the knowledge of lichens and lichecolous fungi of western Ukraine. Polish Botanical Journal 50(1), 39-64.

Boiko TO. 2008 - The lichens and lichenicolous fungi of limestone outcrops of the nature reserve «Yelanetsky step». Chornomorski Botanical Journal 4(1), 84-88.

Boiko TO. 2010 - First data on lichens and lichenicolous fungi of «Pryinhulskiy» Regional Landscape Park (Mycolaiv region). Visnyk of the Lviv University, Series Biology 54, 165171.

Boiko TO. 2013 - The lichen biota of Elanetsko-Inguletskiy region. PhD thesis. Kyiv: M.H. Kholodny Institute of Botany.

Boiko TO, Khodosovtsev AYe. 2011 - New for Ukraine species of lichenicolous fungi from the Natire Reserve «Yelanetskiy Step». Ukrainian Botanical Journal 68(2), 254-258.

Braun U, Khodosovtsev AYe, Darmostuk VV, Diederich P. 2016 - Trichoconis hafellneri sp. nov. on Xanthoria parietina and Athallia pyracea, a generic discussion of Trichoconis and keys to the species of this genus. Herzogia 29(2), 307-314.

Chernov VK. 1895 - About lichens of the city of Kharkov and its environs. Proceedings of the Society of Naturalists at Kharkov University 28, 209-249.

Coppins BJ, Kondratyuk SYa, Khodosovtsev AYe, Zelenko SD et al. 1998 - Diversity of Lichens and Bryophytes in Regional Landscape Park «Stuzhytzia» (Ukrainian part of the International Biosphere Reserve «Eastern Carpathians»): 139-161. In: Kondratyuk SYa, Coppins BJ. 1998 - Lobarion lichens as indicators of the primeval forests of the Eastern Carpathians (Darwin International Workshop, 25-30 May 1998, Kostrino, Ukraine), Kostrino.

Coppins B, Kondratyuk S, Khodosovtsev A, Wolseley P et al. 2001 - New for Crimea and Ukraine species of the lichens. Ukrainian Botanical Journal 58(6), 716-722.

Darmostuk VV. 2015a - Codonmyces lecanorae Calat. \& Etayo is a new species of lichenicolous fungi for Ukraine. Chornomorski Botanical Journal 11(3), 327-329.

Darmostuk VV. 2015b - The first data about lichen biota of Regional Landscape Park «Polovetskyy step». Biological research-2015 1, 24-27.

Darmostuk VV. 2016a - Lichens and lichenicolous fungi of projected reserve tracts «Virovchyna balka» (Kherson). Nauka i metodyka 1: 16-19.

Darmostuk VV. 2016b - Lichenicolous fungi growing on Lecanoraceae in Kherson region. Almanach Qvestiones naturales 6, 201-206.

Darmostuk VV. 2016c - The genus Cercidospora (Dothideales) in Ukraine. Ukrainian Botanical Journal 73(3), 262-267.

Darmostuk VV. 2016d - Lichenicolous mycobiota of National Nature Park «Biloberezhzhya Svyatoslava»: 31. In: Material of International Conference «Advances in botany and ecology (29 June - 3 July 2016, Kherson, Ukraine)», Kherson.

Darmostuk VV. 2016e - Lichen and lichenicolous fungi of reserved tracts «Nedohirskyy forest» (Velykooleksandrivka district, Kherson region). Visnyk of Odessa National University. Biology 21(38), 43-49.

Darmostuk VV. 2016f - Lichens and lichenicolous fungi of the Rusova beam (Velykooleksandrivka district, Kherson region). Studia Biologica 10(2), 133-140.

Darmostuk VV. 2017 - First records of Cercidospora macrospora (Uloth) Hafellner \& Nav.-Ros. anamorph stage: 11-12. In: Material of International Conference «Advances in botany and ecology (5-10 September 2017, Lutsk, Ukraine)», Lutsk. 
Darmostuk VV, Gavrylenko LM. 2016 - Stigmidium gyrophorarum (Arnold) D. Hawksw. is a new lichenicolous fungi for Ukraine. Studia Biologica 10(3-4), 175-179.

Darmostuk VV, Golovenko YeO. 2016 - Polycoccum aksoyi Halıc1 \& V. Atienza is a new species of lichenicolous fungi for Ukraine. Visnyk of Zaporizhzhya National University. Biological Sciences 1, 123-127.

Darmostuk VV, Khodosovtsev AYe. 2014 - Lichens and lichenicolous fungi Kalmius department of Ukrainian Steppe Reserve. Chornomorski Botanical Journal 10(3), 322-327.

Darmostuk VV, Naumovich GO. 2016 - Unguiculariopsis (Helotiaceae, Helotiales), a new genus for the mycobiota of Ukraine. Ukrainian Botanical Journal 73(4), 378-381.

Ertz D, Diederich P, Lawrey JD, Berger F et al. 2015 - Phylogenetic insights resolve Dacampiaceae (Pleosporales) as polyphyletic: Didymocyrtis (Pleosporales, Phaeosphaeriaceae) with Phoma-like anamorphs resurrected and segregated from Polycoccum (Trypetheliales, Polycoccaceae fam. nov.). Fungal Diversity 74(1), 53-89.

Fedorenko NM. 2006 - New and rare lichenicolous fungi of Ukraine. Ukrainian Botanical Journal 63(2), 190-195.

Fedorenko OV, Akulov OYu. 2002 - Materials to study of lichen biota of Mountain Demerdji massiv: 517-520. In: Material of International Conference «Mountains and people», Rahiv.

Fedorenko NM, Kondratyuk SYa, Orlov OO. 2006 - Lichen-forming and lichenicolous fungi of Zhytomyr region. Zhytomyr: P.P. Ruta, Volyn Publichers.

Fedorenko NM, Nadeina OV, Kondratyuk SYa. 2007 - New and rare lichenicolous fungi from Ukraine. Ukrainian Botanical Journal 64(1), 47-56.

Flakus A, Ahti T, Kukwa M, Wilk K. 2008 - New and interesting records of Cladonia and their lichenicolous fungi from the Andean cloud forest in Bolivia. Annales Botanici Fennici 45, 448-454.

Fleischhacker A, Grube M, Frisch A, Obermayer W et al. 2016 - Arthonia parietinaria - A common but frequently misunderstood lichenicolous fungus on species of the Xanthoria parietina-group. Fungal biology 120, 1341-1353.

Gavrylenko LM. 2010 - The lichens of the landscape reserve «Kayirska balka» (Kherson region, Gornostayevskiy district). Science Bulleten of Uzhgorod University (Ser. Biology) 28, 5860 .

Gavrylenko LM. 2011 - The lichen biota of Dudchany balka (Novovorontsovka district, Kherson region), 25-27. In: Material of International Conference «Advances in botany and ecology (1-4 February 2011, Kharkov, Ukraine)», Kharkov.

Gavrylenko LM. 2012 - New for Ukraine lichens and lichenicolous fungi from the Lower Dnieper. Ukrainian Botanical Journal 69(5), 717-720.

Gavrylenko LM. 2014 - The study of lichen species diversity in protected areas ravine-beam system of Lower Dnieper, 84-86. In: Material of International Conference «Regional aspects of floral and faunal studies (10-12 April, Hotyn, Ukraine)», Hotyn.

Gavrylenko LM, Khodosovtsev AYe. 2009 - Lichens and lichenicolous fungi of the Burgunska balka (Khersonska oblast). Chornomorski Botanical Journal 5(1), 28-36.

Gavrylenko LM, Khodosovtsev AYe, Naumovich GO. 2009 - Marchandiobasidium aurantiacum (Lasch) Diederich \& Schultheis - new for Ukraine lichenicolous fungus. Chornomorski Botanical Journal 5(4), 609-611.

Geobotanichne rajonuvannya Ukrainskoy RSR. 1977 - Kiev: Naukova dumka.

Gizhytska ZK. 1929 - Material to mycobiota of Ukraine. Bulletin of the Kiev Botanic Garden 10, 4-41.

Gromakova AB. 2014 - New and rare lichens and lichenicolous fungi for the Left-Bank part of Ukraine from the Seversky Donets River basin. Chornomorski Botanical Journal 10(4), 506514.

Hafellner J. 1985 - Studien über lichenicole Pilze und Flechten III. Die Gattung Roselliniella Vainio emend. Haf. (Ascomycotina, Dothideales). Herzogia 7, 166-169. 
Hawksworth DL. 1992 - Nine lichenicolous fungi from Transcarpathians new for Ukraine. Ukrainian Botanical Journal 49(3), 99-101.

Kapetz NV. 2016 - New and rare to Ukraine lichenicolous fungi. Ukrainian Botanical Journal 73(1), 90-92.

Kapetz NV. 2017 - Lichenicolous fungi of The Teteriv River Basin: 14. In: Material of International Conference «Advances in botany and ecology (5-10 September 2017, Lutsk, Ukraine)», Lutsk.

Kapetz NV, Pleskach LYu, Popova LP, Fedorenko NM et al. 2015 - New to Ukraine and rare species of lichen-forming and lichenicolous fungi. Ukrainian Botanical Journal 72(2), 156163.

Khodosovtsev AYe. 1999a - The lichens of the Black Sea steppes. Kiev: Phytosociocentre.

Khodosovtsev AYe. 1999b - Lichens. Biological and landscape diversity of Crimea: problems and prospects. Issues of the development of the Crimea 11, 22.

Khodosovtsev AYe. 2000 - A new for Crimea and Ukraine species of the lichens. Ukrainian Botanical Journal 57(5), 612-615.

Khodosovtsev AYe. 2002 - A new for Ukraine and Crimea Peninsula species of lichens from Crimean Yaila. Ukrainian Botanical Journal 59(2), 171-178.

Khodosovtsev AYe. 2003 - An annotated list of the lichen forming fungi of the Karadag natural reserve. News of Biosphere Reserve «Askania-Nova» 5, 31-43.

Khodosovtsev AYe. 2004 - The lichens on rock outcrops of Crimea Peninsula. DSc thesis. Kyiv: M.H. Kholodny Institute of Botany.

Khodosovtsev AYe. 2006a - An annotated list of the lichens of the Opukskiy nature reserve. Trudy Nikitskogo botanicheskogo sada. Natsionalnogo nauchogo centra 126, 89-94.

Khodosovtsev AYe. 2006b - An annotated list of the lichens of the Kazantipskiy nature reserve. Trudy Nikitskogo botanicheskogo sada. Natsionalnogo nauchogo centra 126, 216-221.

Khodosovtsev AYe. 2006c - New for Ukraine species of lichens from Crimea. Ukrainian Botanical Journal 63(2), 196-202.

Khodosovtsev AYe. 2006d - New for Crimean Peninsula species of lichens. Chornomorski Botanical Journal 2(1), 98-103.

Khodosovtsev AYe. 2010 - Pyrenochaeta xanthoriae Diederich - a new for Ukraine lichenicolous fungus. Chornomorski Botanical Journal 6(2), 280-281.

Khodosovtsev AYe. 2011 - A new for Ukraine species of the lichenicolous fungi. Chornomorski Botanical Journal 7(2), 194-198.

Khodosovtsev AYe. 2012 - An annotated list of lichenized and lichenicolous fungi of Black Sea Biosphere Reserve. Chornomorski Botanical Journal 8(4), 393-400.

Khodosovtsev AYe. 2013 - Lichen-forming and lichenicolous fungi from Aju-Dag Mt new to Ukraine and Crimea. Chornomorski Botanical Journal 9(1), 84-88.

Khodosovtsev AYe. 2015 - Endocarpo-Xanthocarpion tominii all. nov. and Caloplacetum albolutescentis ass. nov., a new syntaxa of lichen communities from loess outcrops in southern Ukraine. Chornomorski Botanical Journal 11(3), 317-326.

Khodosovtsev AYe, Bogdan OV. 2005 - An annotated catalogue of the lichen forming fungi of the Yalta Mountain-Forest Reserve. Chornomorski Botanical Journal 1(1), 117-132.

Khodosovtsev AYe, Darmostuk VV. 2016a - New species of lichenicolous fungi for Ukraine. Folia Cryptogamica Estonica 53, 93-99.

Khodosovtsev AYe, Darmostuk VV. 2016b - Pleospora xanthoriae sp. nov. (Pleosporaceae, Pleosporales), a new lichenicolous fungus on Xanthoria parietina from Ukraine, with a key to the known lichenicolous species of Dacampia and Pleospora. Opuscula Philolichenum 15, 611 .

Khodosovtsev AYe, Darmostuk VV. 2017a - New species of lichenicolous fungi for Ukraine. U Ukrainian Botanical Journal 74(2), 177-183.

Khodosovtsev AYe, Darmostuk VV. 2017b - Lichens and lichenicolous fungi of granite outcrops of the Bobrynets raivne. Chornomorski Botanical Journal 13(2), 195-203. 
Khodosovtsev AYe, Darmostuk VV. 2017c - Zwackhiomyces polischukii sp. nov., and other noteworthy lichenicolous fungi from Ukraine. Polish Botanical Journal 62(1), 27-35.

Khodosovtsev AYe, Khodosovtseva YuA. 2014 - Lichens and lichenicolous fungi of the arboretum F.E. Falz-Fein Biosphere Reserve of «Askania-Nova». Chornomorski Botanical Journal 10(4), 515-526.

Khodosovtsev AYe, Khodosovtseva YuA. 2015 - The lichens and lichenicolous fungi of National Nature Park «Oleshkivski pisky» (Kherson region, Ukraine). Chornomorski Botanical Journal 11(1), 51-56.

Khodosovtsev AYe, Klymenko VM. 2015 - Didymellopsis perigena (Nyl.) Grube and Zwackhiomyces cervinae Calat., Triebel \& Pérez-Ortega (Xanthopyreniaceae, Ascomycota) new for Ukraine species of the lichenicolous fungi. Chornomorski Botanical Journal 11(2), 217-222.

Khodosovtsev AYe, Marsak II. 2003 - Biatoropsis Ras. - new genus of lichenicolous fungi to mycobiota of Ukraine, 91. In: Material of Ukrainian Conference «Actual issues of modern natural science 2003 (11-13 April, Simferopol, Ukraine)», Simferopol.

Khodosovtsev AYe, Redchenko OO. 2002 - An annotated list of the lichen forming fungi of the natural reserve «Mys Martyan» (Ukraine). Ukrainian Botanical Journal 59(1), 64-71.

Khodosovtsev AYe, Umanets OYu. 2009 - Phoma cladoniicola Diederich, Kocourk. \& Etayo, a new for Ukraine lichenicolous fungus from Oleshkivsky Sands. Chornomorski Botanical Journal 5(2), 273-275.

Khodosovtsev AYe, Zavyalova TV. 2008a - The lichen-forming and lichenicolous fungi of the geological nature monument «Kamyana Mogyla» (Zaporizka oblast, Melitopolskiy district). Chornomorski Botanical Journal 4(2), 264-272.

Khodosovtsev AYe, Zavyalova TV. 2008b - The lichenological zoning of the rocks outcrops of the Kayinkulak river shores (Zaporozhskaya oblast, Chernigovsky district). Visnyk of Odessa National University. Biology 13(16), 56-60.

Khodosovtsev AYe, Darmostuk VV, Gromakova AB. 2016b - New for Ukraine lichen-forming and lichenicolous fungi from Gorgany Nature Reserve. Ukrainian Botanical Journal 73(3), 273-276.

Khodosovtsev AYe, Darmostuk VV, Nazarchuk YuS. 2016d - Lichens and lichenicolous fungi of the Regional Landscape Park «Tiligulskiy» (Odessa region, Ukraine). Chornomorski Botanical Journal 12(2), 165-177.

Khodosovtsev AYe, Gavrylenko LM, Klymenko VM. 2016e - Katherinomyces cetrariae gen. et sp. nov. (asexual Ascomycota) and Sphaerellothecium aculeatae sp. nov. (Mycosphaerellaceae), new lichenicolous fungi on Cetraria aculeata in Ukraine. Nova Hedwigia 102(3-4), 47-55.

Khodosovtsev AYe, Nadyeina OV, Gromakova AB. 2013 - An annotated list of lichen-forming and lichenicolous fungi of Kamyani Mogily Reserve (Ukraine). Chornomorski Botanical Journal 9(4), 542-552.

Khodosovtsev AYe, Vondrak J, Šoun J. 2007 - New lichenized and lichenicolous fungi for the Crimean peninsula (Ukraine). Chornomorski Botanical Journal 3(2), 109-118.

Khodosovtsev AYe, Darmostuk VV, Gromakova AB, Shpilchak MB. 2016c - A first contribution to lichens and lichenicolous fungi of the Nature Reserve «Gorgany» (Ukraine). Chornomorski Botanical Journal 12(1), 51-63.

Khodosovtsev A, Naumovich G, Elix J, Kondratyuk S. 2009 - Lecanora panticapaensis sp. nova and Buelliella poetshii, two noteworthy species from Ukraine. Bibliotheca Lichenologica 100, 189-197.

Khodosovtsev A, Vondrák J, Naumovich A, Kocourková J, Vondráková O, Motiejūnaitė J. 2012 Three new Pronectria species in terricolous and saxicolous microlichen communities (Bionectriaceae, Ascomycota). Nova Hedwigia 95(1-2), 211-220. 
Khodosovtsev A, Dymytrova L, Nadyeina A, Naumovych G et al. 2013b - A contribution to beech forest-associated epiphytic lichen-forming and lichenicolous fungi in Crimean Mts (Ukraine). Flora Mediterranea 23, 57-68.

Kondratyuk SYa. 2005 - New for mycobiota of Ukraine and rare species of Lichenostigma Haf. (Arthoniales, Ascomycotina). Ukrainian Botanical Journal 62(4), 509-516.

Kondratyuk SYa. 2010 - Genus 160. - Buellia De Not. In: Oxner AM. 2010 - Lichen flora of Ukraine. K.: Nauk. dumka.

Kondratyuk SYa. 2012 - Lichens of main habitats of «Hutsulshchyna» National Nature Park. Ukrainian Botanical Journal 69(3), 397-405.

Kondratyuk SYa, Galloway DJ. 1995 - Some new species of lichenicolous fungi. Bibliotheca Lichenologica 58, 235-244.

Kondratyuk SYa, Khodosovtsev AYe. 1997 - New for Ukraine species of lichenicolous fungi. Ukrainian Botanical Journal 54(6), 564-569.

Kondratyuk SYa, Kolomiyets IV. 1997 - New for Ukraine species of lichens and lichenicolous fungi of Reserve «Medobory». Ukrainian Botanical Journal 54(1), 42-47.

Kondratyuk SYa, Andrianova TV, Tykhonenko YuYu. 1999 - Study of mycobiota diversity of Ukraine (Lichenicolous, Septoria and Puccinia fungi). M.G. Kholodny Institute of Botany. Kyiv: Phytosociocentre.

Kondratyuk SYa, Khodosovtsev AYe, Kärnefelt I. 2006 - Llimoniella caloplacae sp. nova (Leothiales), a new lichenicolous fungus on Caloplaca borysthenica sp. nova (Lecanorales, Ascomycota). Mycologica Balcanica 3, 95-99.

Kondratyuk SYa, Khodosovtsev AYe, Zelenko SD. 1998 - The second checklist of lichen forming, lichenicolous and allied fungi of Ukraine. Kiev: Phytosociocentre.

Kondratyuk SYa, Lökös L, Hur J-S. 2014 - New lichen-forming and lichenicolous fungi from Ukraine. Acta Botanica Hungarica 56, 361-368.

Kondratyuk S, Navrotskaya I, Khodosovtsev A, Solonina. O. 1996 - Checklist of Ukrainian lichens. Bocconea 6, 217-294.

Kondratyuk SYa, Popova LP, Lackovicova A, Pišút I. 2003 - A catalogue of the Eastern Carpathian Lichens. Kiev-Bratislava: M.H. Kholodny Institute of Botany.

Kondratyuk SYa, Solonina EF, Navrotskaya IL, Baĭrak EN et al. 1989 - Annotated List of Lichens of the Forest-Steppe Zone of the Ukrainian SSR. Kiev: M.H. Kholodny Institute of Botany.

Kondratyuk SYa, Coppins BJ, Zelenko SD, Khodosovtsev AYe et al. 1998 - Lobarion lichens as indicators of primeval forests in the Ukrainian part of the proposed trilateral reserve «Eastern Carpathians», 64-79. In: Kondratyuk SYa, Coppins BJ. 1998 - Lobarion lichens as indicators of the primeval forests of the Eastern Carpathians (Darwin International Workshop, 25-30 May 1998, Kostrino, Ukraine), Kostrino.

Kopachevskaya YeG. 1986 - Lichenflora of Crimea and their analysis. Kyiv: Nauk. dumka.

Kukwa M. 2000 - Some new and noteworthy lichenicolous fungi to Ukraine. Fragmenta Floristica et Geobotanica 1(45): 532-534.

Lawrey JD, Diederich P, Nelsen MP, Sikaroodi M et al. 2011 - The obligately lichenicolous genus Lichenoconium represents a novel lineage in the Dothideomycetes. Fungal biology 115(2), 176-187.

Makarevich MF. 1955 - To study of lichen flora of Chernivtzi oblast. Botanical Zhurnal of Academy of Sciences of USSR 12(2), 52-59.

Miadlikowska J. 1996 - Contribution to the flora of lichenized Ascomycotina of the Czywczyn Mts. (Eastern Carpathians, Ukraine) III. Peltigera and peltigericolous fungi. Herzogia 12, $129-132$.

Mikhailyuk TI, Kondratyuk SYa, Nyporko SO, Darienko TM et al. 2011 - Lichen-forming fungi, bryophytes and terrestrial algae of granitic canyons of Ukraine. K.: Alterpres.

Morochkovskiy SF, Zerova MYa, Lavitska ZG, Smitska MF. 1969 - Key to the fungi of Ukraine. Ascomycetes. Kyiv: Nauk. dumka. 
Motiejūnaite J, Zalewska A, Kukwa M, Fałtynowicz W. 1999 - New for Ukraine or interesting lichens and allied fungi from the Regional Landscape Park «Stuzhytzia». Ukrainian Botanical Journal 56(6), 596-600.

Nadyeina OV. 2005 - New and rare for Ukraine species of lichens and lichenicolous fungi from Kam'iani Mogyly Reserve. Advances in botany and ecology 1, 15-20.

Nadyeina OV. 2009 - The lichen-forming and lichenicolous fungi of the Donetsk Upland. Mycologia Balcanica 6, 37-53.

Nadyeina O, Halıcı M. 2012 - New lichenicolous fungi records for Kyrgyzstan, Uzbekistan, and Ukraine. Mycotaxon 118(1), 131-136.

Naumovich AO. 2009a - A new and rare for the plain part of Ukraine species of the lichens and lichenicolous fungus from the Ingilets valley. Chornomorski Botanical Journal 5(2), 265-272.

Naumovich AO. 2009b - Lichens of the geological nature monument «Skeli Modru» (Kryvyi Rig city). Chornomorski Botanical Journal 5(3), 442-447.

Naumovich AO, Darmostuk VV. 2015 - Lichenicolous fungi of the valley of Ingulets river (Ukraine). Chornomorski Botanical Journal 11(4), 512-520.

Navrotskaya IL, Kondratyuk SYa, Wasser SP, Nevo E et al. 1996 - Lichens and lichenicolous fungi new for Israel and other countries. Israel Journal of Plant Sciences 44(2-3), 181-193.

Oxner AM. 1930 - New and little known lichen species to the USSR. Visnyk Kyivskogo Botananichnogo Sadu 11, 56-68.

Oxner AM. 1956 - Lichen flora of Ukraine. Kyiv: Vyd-vo AN URSR.

Oxner AM. 2010 - Lichen flora of Ukraine. V. 2. T. 3. Kyiv: Nauk. dumka.

Pirogov MV. 2010a - Clypeococcum hypocenomycis D. Hawksw. - a new species of lichenicolous fungi for Ukraine. Chornomorski Botanical Journal 6(2), 276-279.

Pirogov MV. 2010b - New lichenicolous fungi of Ukrainian Roztochya. Studia Biologica 4(1), $161-164$.

Pirogov MV. 2011 - Nectriopsis rubefaciens (Ellis \& Everh.) M.S. Cole \& D. Hawksw. (Bionectriaceae) - a new species of lichenicolous fungi for Ukraine. Ukrainian Botanical Journal 68(1), 127-128.

Pirogov MV. 2012a - Lichenicolous fungi of the Ukrainian Roztochya. Visnyk of the Lviv University, Series Biology 59, 73-81.

Pirogov MV. 2012b - Two new for mycobiota of Ukraine species lichenicolous fungi from families Nectriaceae and Bionectriaceae (Hypocreales, Ascomycota). Studia Biologica 6(1), 5-19.

Pirogov MV. 2013 - Lichens and lichenicolous fungi of Shatsk National Nature Park (Ukraine). Zarządzanie Ochroną przyrody w lasach 7, 94-108.

Pirogov MV. 2014 - Ascomata micromorphology of Pyrenidium actinellum s. 1. (Dacampiaceae, Ascomycota). Modern Phytomorphology 5, 275-278.

Pirogov MV. 2015 - Clypeococcum cetrariae (Dacampiaceae, Ascomycota) in the Ukrainian Carpathians. Ukrainian Botanical Journal 72(6), 585-587.

Pirogov M, Chepelevska N. 2013 - Cercidospora epipolytropa (Mudd) Arnold in the Ukrainian Carpathians. Visnyk of the Lviv University. Series Biology 61, 37-40.

Pirogov M, Chepelevska N. 2015 - Porpidia tuberculosa та Endococcus brachysporus in Ukrainian Carpathian, 39-40. In: Material of VI opened congress of fitobiologist of Black Sea region (19 May 2015, Kherson-Lazurne, Ukraine), Kherson.

Pirogov MV, Khodosovtsev AYe. 2013 - Lichenicolous fungi Arthonia phaeophysciae Grube \& Matzer (Arthoniaceae) and Taeniolella phaeophysciae D. Hawksw. (Anamorphic Ascomycota), new species of Ukraine. Ukrainian Botanical Journal 70(4), 535-537.

Pirogov MV, Sharavara SM. 2012 - Phaeospora rimosicola (Verrucariaceae, Ascomycota) - new species of lichenicolous fungi for Ukraine. Studia Biologica 6(1), 201-204.

Pirogov MV, Sharavara SM. 2013 - Vouauxiella lichenicola (Linds.) Petrak \& Sydow, a rare species of lichenicolous fungi in Western Ukraine. Ukrainian Botanical Journal 70(5), 681682. 
Pirogov M Shovhan Yu. 2015 - Notes on the lichen biota of National Nature Park «Pivnichne Podilla». Visnyk of the Lviv University. Series Biology 69, 93-101.

Pirogov M, Chepelevska N, Vondrák J. 2014 - Carbonea in Ukraine. Studia Biologica 8(1), 137148.

Pirogov M, Tasenkevich L, Szaravara S. 2015 - Notatki o biocie epilitycznych i epigeicznych porostów Parku Narodowego Beskidy Skolskie. Roczniki Bieszczadzkie 23, 81-93.

Prekrasna Ye, Vasylyuk O, Domashevskyy S, Parnikoza I et al. 2012 - The projected of National Nature Park «Divychky» in the Kiev region. Kyiv: National Ecological Center of Ukraine.

Raduka LP, Zelenko SD. 1998 - Lobaria pulmonaria (L.) Hoffm. in the «Synevyr» National Nature Park (Ukrainian Carpathians), 117-119. In: Kondratyuk SYa, Coppins BJ. 1998 Lobarion lichens as indicators of the primeval forests of the Eastern Carpathians (Darwin International Workshop, 25-30 May 1998, Kostrino, Ukraine), Kostrino.

Redchenko AA. 2001 - New and rare species of lichens from coastal Crimea. Ukrainian Botanical Journal 58(5), 578-582.

Roux C, Triebel D. 1994 - Revision des especes de Stigmidium et de Sphaerellothecium (champignons lichenicoles non lichenises, Ascomycetes) correspondant a Pharcidia epicymatia sensu Keissler ou a Stigmidium schaereri auct. Bulletin de la Societe Lineenne de Provence 45, 451-542.

Rusina NV, Nadyeina OV, Khodosovtsev AYe. 2010 - An annotated list of lichen-forming and lichenicolous fungi of Lugans'ky natural reserve. Chornomorski Botanical Journal 4(1), 247 258.

Servít M. 1936 - Neue und seltenere Flechten aus den Familien Verrucariaceae und Dermatocarpaceae. Beihefte zum Bot. Centralbl. 55, 251-274.

Servít M, Nádvorník J. 1932 - Flechten aus der Čechoslovakei. II. Karpatorussland und Sudostslovakei. Věstn. Král. Čes. Spol. Nauk., Třr. Mat.-Přír., 1931-1932, 1-41.

Smerechynska TO. 2006 - Lichens of the Medobory Nature Reserve. PhD thesis. Kyiv: M.H. Kholodny Institute of Botany.

Vondrák J, Šoun J. 2007 - Lichenostigma svandae, a new lichenicolous fungus on Acarospora cervina. Lichenologist 39(3), 211-216.

Vondrák J, Palice Z, Khodosovtsev A, Postoyalkin S. 2010 - Additions to the diversity of rare or overlooked lichens and lichenicolous fungi in Ukrainian Carpathians. Chornomorski Botanical Journal 6(1), 6-34.

Zavyalova TV. 2010 - Lichens and lichenicolous fungi of the «Staroberdyansky» and «Altagirsky» forests (South Ukraine). Chornomorski Botanical Journal 6(3), 400-403.

Zelenko SD. 2005 - Toninia talparum (Lecideaceae, Ascomycetes) - new species for lichenobiota Eurasia from Podoliya Heights. Ukrainian Botanical Journal 62(4), 517-521. 